Article

\title{
On the Basel Liquidity Formula for Elliptical Distributions
}

\author{
Janine Balter ${ }^{1, \dagger}$ and Alexander J. McNeil ${ }^{2, * \mathbb{C}}$ \\ 1 Deutsche Bundesbank, 40212 Düsseldorf, Germany; janine.balter@york.ac.uk \\ 2 The York Management School, University of York, Freboys Lane, York YO10 5GD, UK \\ * Correspondence: alexander.mcneil@york.ac.uk; Tel.: +44-1904-325307 \\ + The opinions expressed in this paper are those of the author and do not necessarily reflect views shared by \\ the Deutsche Bundesbank or its staff.
}

Received: 5 July 2018; Accepted: 3 September 2018; Published: 7 September 201

\begin{abstract}
A justification of the Basel liquidity formula for risk capital in the trading book is given under the assumption that market risk-factor changes form a Gaussian white noise process over 10-day time steps and changes to P\&L (profit-and-loss) are linear in the risk-factor changes. A generalization of the formula is derived under the more general assumption that risk-factor changes are multivariate elliptical. It is shown that the Basel formula tends to be conservative when the elliptical distributions are from the heavier-tailed generalized hyperbolic family. As a by-product of the analysis, a Fourier approach to calculating expected shortfall for general symmetric loss distributions is developed.
\end{abstract}

Keywords: Basel Accords; liquidity risk; risk measures; expected shortfall; elliptical distributions; generalized hyperbolic distributions

\section{Introduction}

As a result of the fundamental review of the trading book (FRTB) (Basel Committee on Banking Supervision 2013) a new minimum capital standard for the trading book has emerged (Basel Committee on Banking Supervision 2016). Under this standard, banks are now required to calculate a liquidity-adjusted expected shortfall risk measure on a daily basis. This calculation is carried out at both the level of the whole trading book and the level of individual desks using an aggregation formula that is based on the concepts of liquidity horizons and square-root-of-time scaling.

The Basel liquidity formula uses the language of risk factors. These are the fundamental quantities such as asset prices, index values, interest rates and exchange rates that are required to value the various positions in the trading book at any point in time. In addition to prices and rates, the set of risk factors contains a number of market-observable parameters including implied volatilities, which are used as inputs to model-based formulas for the valuation of derivative securities such as options.

Every risk factor is assigned to a unique liquidity bucket $j$ associated with a liquidity horizon $\mathrm{LH}_{j}$ which may be 10,20,40,60 or 120 days. These horizons are conservative estimates of the amount of time that would be required to execute trades that would eliminate the portfolio's sensitivity to changes in these risk factors during a period of market illiquidity. For example, risk factors describing the price risk of large-cap equities are assigned to the bucket with the shortest horizon of 10 days; risk factors describing volatility risk for derivatives involving large-cap equities are given a risk horizon of 20 days; risk factors for structured credit instruments (e.g., collateralized debt obligations) have the longest liquidity horizon of 120 days.

The liquidity formula requires that a series of expected shortfall charges are calculated with respect to 'shocks' to certain risk factors while other risk factors are held constant. The shocks are estimates of the more extreme joint changes in risk-factor values that could occur over a fixed horizon 
of $T$ days. For most banks, what this means in practice is that historical risk-factor changes for the selected risk factors over the horizon $T$ are applied to the positions to obtain a so-called P\&L or profit-and-loss distribution. While this P\&L distribution can be obtained by full revaluation of the positions in the portfolio, most banks use a simpler approach in which they consider only the P\&L resulting from first-order (delta) and possibly second-order (gamma) sensitivities to the risk-factor changes. Having obtained the distribution, the effect of the shock is computed by applying the expected shortfall risk measure.

To make the calculation explicit, we give the formula and notation as published on p. 52 of the revised capital standard (Basel Committee on Banking Supervision 2016).

- let $T=\mathrm{LH}_{1}$ denote the so-called base liquidity horizon of 10 days.

- Let $\operatorname{ES}_{T}(P)$ denote the expected shortfall at horizon $T$ and a $97.5 \%$ confidence level for a portfolio $P$ with respect to shocks to all risk factors to which the positions in the portfolio are exposed.

- $\quad$ Let $\mathrm{ES}_{T}(P, j)$ denote the expected shortfall at horizon $T$ and a $97.5 \%$ confidence level for a portfolio $P$ with respect to shocks to the risk factors which have a liquidity horizon of length $\mathrm{LH}_{j}$ or greater, with all other risk factors held fixed.

The liquidity-adjusted expected shortfall is

$$
\mathrm{ES}=\sqrt{\left(\mathrm{ES}_{T}(P)\right)^{2}+\sum_{j \geq 2}\left(\mathrm{ES}_{T}(P, j) \sqrt{\frac{\mathrm{LH}_{j}-\mathrm{LH}_{j-1}}{T}}\right)^{2}} .
$$

The bank computes the expected shortfall charges $\operatorname{ES}_{T}(P)$ and $\mathrm{ES}_{T}(P, j)$ and evaluates the right-hand side of (1). The resulting number ES is then an important determinant of the bank's overall capital charge for trading activities. (There are a number of further adjustments and add-on charges that we will not go in to.)

The formula is mysterious at first sight but some rough intuition can be gained by observing that the squared capital charge $\mathrm{ES}^{2}$ is given by a sum of terms $\mathrm{ES}_{T}(P, j)^{2}\left(\mathrm{LH}_{j}-\mathrm{LH}_{j-1}\right) / T$ for $j=1, \ldots, 5$, where $\mathrm{ES}_{T}(P)^{2}$ corresponds to $j=1$. The square root of each of these terms can be thought of as measuring the risk contribution arising from position liquidations between $\mathrm{LH}_{j-1}$ and $\mathrm{LH}_{j}$. The scaling factors $\sqrt{\left(\mathrm{LH}_{j}-\mathrm{LH}_{j-1}\right) / T}$ take into account that $\mathrm{ES}_{T}(P, j)$ is an expected shortfall charge calculated over the interval $[0, T]$. They are an example of square-root-of-time scaling which is widely used in finance to translate certain measures of risk (e.g., volatility, value-at-risk and expected shortfall) calculated on shorter time intervals to longer time intervals.

The first objective of this paper is to provide a principles-based derivation of this formula that relates it to the concept of expected shortfall as a risk measure applied to a loss distribution. Most practitioners know that an assumption of normality underlies the formula but exact details are not available in the main regulatory documents in the public domain. We make it precise that the formula can be justified by assuming that risk-factor changes over time steps equal to the base liquidity horizon form a multivariate Gaussian white noise with mean zero and portfolio losses are all attributable to first-order (delta) sensitivities to the risk-factor changes.

The second and major objective of the paper is to extend the formula under the more general assumption that risk-factor changes have a multivariate elliptical distribution. This allows us to consider some particular cases with heavy tails and tail dependencies that might be considered more realistic models for market risk-factor changes.

Many results in quantitative risk management (QRM) continue to hold when multivariate normal assumptions are generalized to multivariate elliptical assumptions. In particular, when losses are linear in a set of underlying elliptically-distributed risk factors, aggregation of risk measures across different business lines, desks or risk factors can generally be based on a common formulaic approach, regardless of the exact choice of elliptical distribution; see Chapter 8 of McNeil et al. (2015). The difference in the current paper is that aggregation takes place, not only across risk factors, but also 
across time and therefore a 'central limit effect' takes place. This means that (1) does not hold in the general elliptical case.

We derive a generalization of (1) that applies to all elliptical distributions. Using this generalization we consider, in particular, a number of the heavier-tailed distributions in the symmetric generalized hyperbolic family (a sub-family of the elliptical distributions). We infer that, for these distributions, the use of the standard aggregation rule in (1) would lead to a conservative capital charge in the sense that the resulting amount of capital ES is larger than is actually necessary to achieve the level of risk targeted by FRTB (expected shortfall at a $97.5 \%$ confidence level).

As a by-product of our analyses we also demonstrate a new approach to calculating VaR and expected shortfall for symmetric distributions with a known characteristic function. This approach is particularly useful in cases where we take convolutions of elliptically distributed random vectors and often lose the ability to write simple closed-form expressions for their probability densities.

We present all ideas in terms of the standard probabilistic approach to risk measures. Losses (or P\&L variables) are represented by random variables $L$. Expected shortfall $\left(\mathrm{ES}_{\alpha}\right)$ and value-at-risk $\left(\mathrm{VaR}_{\alpha}\right)$ at level $\alpha$ are risk measures applied to $L$. If $F_{L}$ denotes the distribution function of $L$ and $F_{L}^{\leftarrow}$ its generalized inverse, they are given by $\operatorname{VaR}_{\alpha}(L)=F_{L}^{\leftarrow}(\alpha)$ and $\operatorname{ES}_{\alpha}(L)=\frac{1}{1-\alpha} \int_{\alpha}^{1} F_{L}^{\leftarrow}(u) \mathrm{d} u$. If $F_{L}$ is continuous then the formula $\mathrm{ES}_{\alpha}(L)=\mathbb{E}\left(L \mid L \geq \operatorname{VaR}_{\alpha}(L)\right)$ also holds.

\section{Justifying and Extending the Basel Liquidity Formula}

Let $\left(\boldsymbol{X}_{t}\right)$ be a $d$-dimensional time series of risk-factor changes for all relevant risk factors and assume that these are all defined in terms of simple differences or log-differences. We interpret $X_{t+1}$ as the vector of risk-factor changes over the time step $[t, t+1]$. In practice this time step will be equal to the base liquidity horizon of 10 days.

For $h \in \mathbb{N}$, the risk factor changes over the time step $[t, t+h]$ are given additively by

$$
\boldsymbol{X}_{[t, t+h]}:=\sum_{j=1}^{h} \boldsymbol{X}_{t+j}
$$

Without loss of generality let the risk calculation be made at time $t=0$. We make the following assumptions.

\section{Assumption 1.}

(i) The risk-factor changes $\left(\boldsymbol{X}_{t}\right)$ form a stationary white noise process (a serially uncorrelated process) with mean zero and covariance matrix $\Sigma$.

(ii) Each risk factor may be assigned to a unique liquidity bucket $B_{k}$ defined by a liquidity horizon $h_{k} \in \mathbb{N}$, $k=1, \ldots, n$.

(iii) In the event of a portfolio liquidation action the loss (or profit) attributable to risk factors in bucket $B_{k}$ is given by $\boldsymbol{b}_{k}^{\prime} \boldsymbol{X}_{\left[0, h_{k}\right]}$ where $\boldsymbol{b}_{k}$ is a weight vector with zeros in any position that corresponds to a risk factor that is not in $B_{k}$.

Assumption 1(iii) contains the linearity assumption and adopts the pessimistic view that the full liquidity horizon $h_{k}$ is required to remove the portfolio's sensitivity to all the risk factors in liquidity bucket $B_{k}$.

Under these assumptions we compute the portfolio loss $L$ over the maximum time horizon $h_{n}$, which is the time required to remove the portfolio's sensitivity to all risk factors. It follows from Assumption 1(ii) and (iii) that

$$
L=\sum_{k=1}^{n} \boldsymbol{b}_{k}^{\prime} \boldsymbol{X}_{\left[0, h_{k}\right]}=\sum_{k=1}^{n} \sum_{j=1}^{k} \boldsymbol{b}_{k}^{\prime} \boldsymbol{X}_{\left[h_{j-1}, h_{j}\right]}=\sum_{k=1}^{n} \sum_{j=k}^{n} \boldsymbol{b}_{j}^{\prime} \boldsymbol{X}_{\left[h_{k-1}, h_{k}\right]}=\sum_{k=1}^{n} \boldsymbol{\beta}_{k}^{\prime} \boldsymbol{X}_{\left[h_{k-1}, h_{k}\right]}
$$


where $\boldsymbol{\beta}_{k}=\sum_{j=k}^{n} \boldsymbol{b}_{j}$ and $h_{0}=0$. The vector $\boldsymbol{\beta}_{k}$ contains the weights for all risk factors in the union of liquidity buckets $B_{k} \cup \cdots \cup B_{n}$.

Let us write $L_{k}:=\boldsymbol{\beta}_{k}^{\prime} \boldsymbol{X}_{\left[h_{k-1}, h_{k}\right]}$ for $k=1, \ldots, n$ for the summands in the final expression in (3). These are uncorrelated by Assumption 1(i) and we may easily calculate that

$$
\operatorname{var}(L)=\sum_{k=1}^{n} \operatorname{var}\left(L_{k}\right)=\sum_{k=1}^{n} \boldsymbol{\beta}_{k}^{\prime} \operatorname{cov}\left(\boldsymbol{X}_{\left[h_{k-1}, h_{k}\right]}\right) \boldsymbol{\beta}_{k}=\sum_{k=1}^{n}\left(h_{k}-h_{k-1}\right) \boldsymbol{\beta}_{k}^{\prime} \Sigma \boldsymbol{\beta}_{k}
$$

where the final step follows because (2) implies that $\boldsymbol{X}_{\left[h_{k-1}, h_{k}\right]}=\sum_{j=1}^{h_{k}-h_{k-1}} \boldsymbol{X}_{h_{k-1}+j}$.

We now introduce random variables

$$
L^{(k)}=\boldsymbol{\beta}_{k}^{\prime} \boldsymbol{X}_{\left[0, h_{1}\right]}
$$

for $k=1, \ldots, n$. These represent losses attributable to all risk factors in the union of liquidity buckets $B_{k} \cup \cdots \cup B_{n}$ over the liquidity horizon $h_{1}$. Note that the $L_{k}$ and $L^{(k)}$ variables differ (unless $k=1$ ). Since $\operatorname{var}\left(L^{(k)}\right)=h_{1} \boldsymbol{\beta}_{k}^{\prime} \Sigma \boldsymbol{\beta}_{k}$, we obtain from (4) the formula

$$
\operatorname{sd}(L)=\sqrt{\sum_{k=1}^{n}\left(\sqrt{\frac{h_{k}-h_{k-1}}{h_{1}}} \operatorname{sd}\left(L^{(k)}\right)\right)^{2}} .
$$

It may be noted that the presence of positive correlation between the variables $L_{k}$ in (4), caused by serial correlation in the underlying risk-factor changes $\boldsymbol{X}_{\left[h_{k-1}, h_{k}\right]}$, would tend to lead to the left-hand side of (6) being larger than the right-hand side. Negative correlation would lead to it being smaller.

\subsection{The Gaussian Case}

Suppose that $\left(\boldsymbol{X}_{t}\right)$ is a Gaussian process; in this case $\left(\boldsymbol{X}_{t}\right)$ is actually a strict white noise (a process of independent and identically distributed vectors). It follows that $L_{k} \sim N\left(0,\left(h_{k}-h_{k-1}\right) \beta_{k}^{\prime} \Sigma \beta_{k}\right)$ and the $L_{k}$ are independent for all $k$. Thus, by the convolution property for independent normals,

$$
L \sim N\left(0, \sum_{k=1}^{n}\left(h_{k}-h_{k-1}\right) \boldsymbol{\beta}_{k}^{\prime} \Sigma \boldsymbol{\beta}_{k}\right) .
$$

Moreover, we clearly have $L^{(k)} \sim N\left(0, h_{1} \boldsymbol{\beta}_{k}^{\prime} \Sigma \boldsymbol{\beta}_{k}\right)$.

For any mean-zero normal random variable $V$ it is easy to show that $\operatorname{ES}_{\alpha}(V)=c_{\alpha} \operatorname{sd}(V)$ where $c_{\alpha}=\phi\left(\Phi^{-1}(\alpha)\right) /(1-\alpha), \phi$ denotes the density of the standard normal distribution and $\Phi^{-1}(\alpha)$ denotes the $\alpha$-quantile of the standard normal distribution function $\Phi$ (see McNeil et al. 2015, chp. 2). It follows from (6) that

$$
\mathrm{ES}_{\alpha}(L)=\sqrt{\sum_{k=1}^{n}\left(\sqrt{\frac{h_{k}-h_{k-1}}{h_{1}}} \mathrm{ES}_{\alpha}\left(L^{(k)}\right)\right)^{2}}
$$

which is the proposed standard formula for the trading book (1) rewritten in our notation.

\subsection{An Extension to the Formula for Elliptical Distributions}

In this section we assume a centred elliptical distribution for the risk-factor changes, which subsumes the multivariate normal distribution as a special case. The class of elliptical distributions contains a number of particular distributions which are popular models for financial returns including the multivariate Student $t$ and the symmetric generalized hyperbolic distributions. There is much empirical evidence that 10-day and even monthly risk-factor returns of different types are heavier tailed than Gaussian; see, for example, Section 6.2.4 of McNeil et al. (2015). Distributions in the symmetric 
generalized hyperbolic family provide a superior fit, although they do not address the issue of asymmetry which is a feature of certain risk-factor returns such as equity returns.

In simple terms, elliptical distributions are affine transformations of spherical distributions and spherical distributions can be thought of as distributions that are invariant under rotations. More formally, a random vector $Y_{t}$ is said to have a $d$-dimensional spherical distribution with characteristic generator $\psi$, written $Y_{t} \sim S_{d}(\psi)$, if its characteristic function satisfies $\phi(s)=E\left(e^{i s^{\prime} Y_{t}}\right)=\psi\left(\boldsymbol{s}^{\prime} \boldsymbol{s}\right)$ for a function of a scalar variable $\psi$. The covariance matrix of $Y_{t}$ is a scalar multiple of the $d$-dimensional identity matrix $I_{d}$ satisfying $\operatorname{cov}\left(Y_{t}\right)=\operatorname{var}(Y) I_{d}$ where $Y \sim S_{1}(\psi)$ denotes any component of the vector $\boldsymbol{Y}_{t}$.

A random vector $X_{t}$ is said to have a $d$-dimensional elliptical distribution with location vector $\mu$, positive-definite dispersion matrix $\Omega$ and characteristic generator function $\psi$, written $X_{t} \sim E_{d}(\mu, \Omega, \psi)$ if $X_{t}=\mu+A Y_{t}$ for some matrix $A \in \mathbb{R}^{d \times d}$ satisfying $\Omega=A A^{\prime}$ and some spherically distributed random vector $Y_{t} \sim S_{d}(\psi)$. It follows that the covariance matrix of $X_{t}$ is given by $\Sigma=\operatorname{var}(Y) \Omega$, which shows that the covariance matrix is in general a scalar multiple of $\Omega$. See Fang et al. (1990) and McNeil et al. (2015) for further details of these distributions.

In addition to Assumption 1 we assume that the following holds in this section.

\section{Assumption 2.}

(i) The process $\left(\boldsymbol{X}_{t}\right)$ is a multivariate strict white noise (an iid process).

(ii) For every $t, X_{t} \sim E_{d}(\mathbf{0}, \Omega, \psi)$ where $\Omega$ is a positive-definite matrix.

Assumption 2(i) may seem strong but in practice we assume that $\left(\boldsymbol{X}_{t}\right)$ is a process of 10-day returns so that the iid assumption, while unlikely to be true, is less problematic than for daily financial returns. The assumption is required in order to analyse convolutions of elliptically distributed random vectors with different characteristic generators.

We need three key properties of an elliptical distribution for our calculation. Let $X \sim E_{d}(\mathbf{0}, \Omega, \psi)$ and $\tilde{X} \sim E_{d}(\mathbf{0}, \Omega, \tilde{\psi})$ be independent elliptically-distributed variables with the same dispersion matrix $\Omega$ and possibly different characteristic generators $\psi$ and $\tilde{\psi}$.

$$
\begin{array}{ll}
\boldsymbol{\beta}^{\prime} \boldsymbol{X} \sim E_{1}\left(0, \boldsymbol{\beta}^{\prime} \Omega \boldsymbol{\beta}, \psi\right) & \text { for } \boldsymbol{\beta} \in \mathbb{R}^{d} \text { and } \boldsymbol{\beta} \neq \mathbf{0} . \\
\boldsymbol{X} \sim E_{d}(\mathbf{0}, c \Omega, \psi(s / c)) & \text { for any } c>0 . \\
\boldsymbol{X}+\tilde{\boldsymbol{X}} \sim E_{d}\left(\mathbf{0}, \Omega, \psi^{*}\right) & \text { where } \psi^{*}(s)=\psi(s) \tilde{\psi}(s) .
\end{array}
$$

We will use (9) and (11) to find the characteristic functions of elliptical random vectors under linear combinations and convolutions respectively. The property in (10) shows that we have some discretion in how we represent the characteristic generator of an elliptical random variable in terms of its characteristic generator and its scaling.

Theorem 1. Under Assumptions 1 and 2 the loss $L$ in (3) is a univariate spherical random variable $L \sim S_{1}\left(\psi_{L}\right)$ with characteristic generator

$$
\psi_{L}(s)=\prod_{k=1}^{n} \psi_{k}\left(s \boldsymbol{\beta}_{k}^{\prime} \Omega \boldsymbol{\beta}_{k}\right),
$$

where $\psi_{k}=\psi^{h_{k}-h_{k-1}}$ is the $\left(h_{k}-h_{k-1}\right)$-fold product of $\psi$ for $k=1, \ldots, n$.

For $\alpha>0.5$ the expected shortfall of $L$ is related to the expected shortfall of the variables $L^{(k)}$ in (5) by

$$
\mathrm{ES}_{\alpha}(L)=\frac{c_{\alpha, \psi_{L}}}{\mathcal{c}_{\alpha, \psi_{1}}} \sqrt{\sum_{k=1}^{n}\left(\sqrt{\frac{h_{k}-h_{k-1}}{h_{1}}} \mathrm{ES}_{\alpha}\left(L^{(k)}\right)\right)^{2}} .
$$


where $c_{\alpha, \psi_{L}}$ represents the ratio of expected shortfall to standard deviation for $L$ and $c_{\alpha, \psi_{1}}$ is the equivalent ratio for a univariate spherical variable $Z \sim S_{1}\left(\psi_{1}\right)$.

Proof. We need to derive the distributions of

$$
L_{k}=\boldsymbol{\beta}_{k}^{\prime} \boldsymbol{X}_{\left[h_{k-1}, h_{k}\right]}, \quad L=\sum_{k=1}^{n} L_{k} \quad \text { and } \quad L^{(k)}=\boldsymbol{\beta}_{k}^{\prime} \boldsymbol{X}_{\left[0, h_{1}\right]} .
$$

First note that if $\boldsymbol{X}_{t} \sim E_{d}(\mathbf{0}, \Omega, \psi)$ then it follows from (2) and (11) that $\boldsymbol{X}_{\left[h_{k-1}, h_{k}\right]} \sim E_{d}\left(\mathbf{0}, \Omega, \psi_{k}\right)$ where $\psi_{k}=\psi^{h_{k}-h_{k-1}}$. Using (9) we have that

$$
L_{k} \sim E_{1}\left(0, \boldsymbol{\beta}_{k}^{\prime} \Omega \beta_{k}, \psi_{k}\right) \text { and } L^{(k)} \sim E_{1}\left(0, \beta_{k}^{\prime} \Omega \beta_{k}, \psi_{1}\right)
$$

Using (10) we write the former as $L_{k} \sim E_{1}\left(0,1, \psi_{k}\left(s \boldsymbol{\beta}_{k}^{\prime} \Omega \boldsymbol{\beta}_{k}\right)\right)$ or $L_{k} \sim S_{1}\left(\psi_{k}\left(s \boldsymbol{\beta}_{k}^{\prime} \Omega \boldsymbol{\beta}_{k}\right)\right)$ and then use the convolution property (11) to conclude that $L \sim S_{1}\left(\psi_{L}\right)$ where $\psi_{L}$ is given in (12).

Now $\mathrm{ES}_{\alpha}\left(L^{(k)}\right)=\sqrt{\boldsymbol{\beta}_{k}^{\prime} \Omega \boldsymbol{\beta}_{k}} \mathrm{ES}_{\alpha}(Z)$ and $\operatorname{sd}\left(L^{(k)}\right)=\sqrt{\boldsymbol{\beta}_{k}^{\prime} \Omega \boldsymbol{\beta}_{k}} \operatorname{sd}(Z)$ where $Z \sim S_{1}\left(\psi_{1}\right)$. Hence it follows that $\mathrm{ES}_{\alpha}\left(L^{(k)}\right)=c_{\alpha, \psi_{1}} \operatorname{sd}\left(L^{(k)}\right)$ for all $k$ and

$$
\begin{aligned}
\operatorname{ES}_{\alpha}(L)=c_{\alpha, \psi_{L}} \operatorname{sd}(L) & =c_{\alpha, \psi_{L}} \sqrt{\sum_{k=1}^{n}\left(\sqrt{\frac{h_{k}-h_{k-1}}{h_{1}}} \operatorname{sd}\left(L^{(k)}\right)\right)^{2}} \\
& =c_{\alpha, \psi_{L}} \sqrt{\sum_{k=1}^{n}\left(\sqrt{\frac{h_{k}-h_{k-1}}{h_{1}}} \frac{\operatorname{ES}\left(L^{(k)}\right)}{c_{\alpha, \psi_{1}}}\right)^{2}}
\end{aligned}
$$

which yields (13).

It may be easily verified that when $\psi(s)=\exp (-s / 2)$ (the Gaussian case), the characteristic function $\phi(s)=\psi_{L}\left(s^{2}\right)$ implied by (12) is the characteristic function of the normal distribution in (7). In this case the constants $c_{\alpha, \psi_{L}}$ and $c_{\alpha, \psi_{1}}$ are identical.

When the risk factors have a heavier-tailed distribution than normal we expect that $c_{\alpha, \psi_{L}} \leq c_{\alpha, \psi_{1}}$. This is because the aggregation across time periods that takes place in the definition of $L$ should lead to a central limit effect whereby $L$ is closer to Gaussian than the $L^{(k)}$ variables. In this case we expect that the standard Basel liquidity formula should give an upper bound on $\operatorname{ES}_{\alpha}(L)$.

\section{Calculating the Scaling Ratio in Practice}

We turn to the problem of calculating the ratio $r_{\alpha}:=c_{\alpha, \psi_{L}} / c_{\alpha, \psi_{1}}$ when the underlying risk factors have an elliptical distribution with generator $\psi$. To compute $c_{\alpha, \psi_{1}}$ we calculate the ratio $\mathrm{ES}_{\alpha}(Z) / \operatorname{sd}(Z)$ for a univariate spherical random variable $Z$ with characteristic generator $\psi_{1}=\psi^{h_{1}}$. To compute $c_{\alpha, \psi_{L}}$ we calculate the ratio $\mathrm{ES}_{\alpha}(L) / \mathrm{sd}(L)$ for a univariate spherical variable $L$ with characteristic generator given by (12).

The problem of calculating expected shortfall for linear portfolios of elliptically distributed risk factors is tackled in Kamdem (2005) and Dobrev et al. (2017). The proposed method relies on knowing the so-called density generator of the elliptical distribution. In our application the taking of convolutions means that the density generator required to calculate $\mathrm{ES}_{\alpha}(Z)$ and $\mathrm{ES}_{\alpha}(L)$ may not be available in a simple closed forms for the underlying distributions of $X_{t}$ which interest us.

However, the characteristic generator is always available in our application. In the following section we give results that can be used to compute expected shortfall directly from the characteristic function of a spherical random variable. 


\subsection{Calculating Expected Shortfall by Fourier Inversion}

A univariate spherical random variable $Y \sim S_{1}(\psi)$ is symmetric about the origin with a real-valued even characteristic function given by $\phi_{Y}(s):=\psi\left(s^{2}\right)$. We give a general result that applies to univariate random variables that are symmetric about the origin.

Theorem 2. Let $Y$ be symmetrically distributed about the origin with an integrable characteristic function $\phi_{Y}(s)$. Let $-\infty<a<b<\infty$. Then the following formulas hold:

$$
\begin{aligned}
f_{Y}(y) & =\frac{1}{\pi} \int_{0}^{\infty} \cos (s y) \phi_{Y}(s) \mathrm{d} s, \\
F_{Y}(y) & =\frac{1}{2}+\frac{1}{\pi} \int_{0}^{\infty} \frac{\sin (s y)}{s} \phi_{Y}(s) \mathrm{d} s \\
E\left(Y I_{\{a \leq Y \leq b\}}\right) & =\frac{1}{\pi} \int_{0}^{\infty} \frac{b s \sin (b s)+\cos (b s)-a s \sin (a s)-\cos (a s)}{s^{2}} \phi_{Y}(s) \mathrm{d} s .
\end{aligned}
$$

Proof. The characteristic function $\phi_{Y}(s)$ of a random variables that is symmetric about the origin is real-valued and even. If $\phi_{Y}$ is integrable then the density exists and the standard Fourier inversion formula for the characteristic formula yields

$$
f_{Y}(y)=\frac{1}{2 \pi} \int_{-\infty}^{\infty} e^{-i s y} \phi_{Y}(s) \mathrm{d} s=\frac{1}{\pi} \int_{0}^{\infty} \cos (s y) \phi_{Y}(s) \mathrm{d} s .
$$

The formula (16) for the distribution function is obtained from a well-known representation by Gil-Pelaez (1951). To derive (17) we observe that

$$
\begin{aligned}
\int_{a}^{b} y f_{Y}(y) & =\frac{1}{\pi} \int_{a}^{b} \int_{0}^{\infty} y \cos (s y) \phi_{Y}(s) \mathrm{d} s \mathrm{~d} y \\
& =\frac{1}{\pi} \int_{0}^{\infty}\left(\int_{a}^{b} y \cos (s y) \mathrm{d} y\right) \phi_{Y}(s) \mathrm{d} s
\end{aligned}
$$

by Fubini's Theorem since $\left|y \cos (s y) \phi_{Y}(s)\right| \leq|y|\left|\phi_{Y}(s)\right|$ and the latter is integrable on $[a, b] \times[0, \infty)$. The inner integral can be solved by parts to obtain

$$
\int_{a}^{b} y \cos (s y) \mathrm{d} y=\frac{b s \sin (b s)+\cos (b s)-a s \sin (a s)-\cos (a s)}{s^{2}}
$$

and (17) follows.

These formulas permit the accurate evaluation of $\operatorname{VaR}_{\alpha}(Y)$ and expected shortfall using one-dimensional integration. Calculation of $\operatorname{VaR}_{\alpha}(Y)$ for $\alpha>0.5$ is accomplished by numerical root finding using (16). If $\mathbb{E}|Y|<\infty$ for the distribution in question, then expected shortfall is defined and it can be calculated by setting $a=\operatorname{VaR}_{\alpha}(Y)$ and computing the limit

$$
\mathrm{ES}_{\alpha}(Y)=\lim _{b \rightarrow \infty} \frac{1}{\pi(1-\alpha)} \int_{0}^{\infty} \frac{b s \sin (b s)+\cos (b s)-a s \sin (a s)-\cos (a s)}{s^{2}} \phi_{Y}(s) \mathrm{d} s .
$$

Our experiments confirm that calculating the integral in (18) for increasing $b$ does result in stable limiting values for $\operatorname{ES}_{\alpha}(Y)$ which agree to a high level of accuracy with theoretical values for well-known distributions such as Student $\mathrm{t}$.

\subsection{The Case of Generalized Hyperbolic Distributions}

We will apply Theorem 2 to the family of symmetric generalized hyperbolic (GH) distributions. This is a very popular family for modelling financial returns and there are many useful sources for the properties of these distributions including Barndorff-Nielsen (1978); Barndorff-Nielsen and Blæsild (1981); 
Eberlein (2010) and McNeil et al. (2015). While some special cases of the GH family are known to be invariant under convolutions (Podgórski and Wallin 2016) the complicated aggregation of variables with different scaling that we undertake means that, even for these cases, we generally need to use (18) to compute expected shortfall for the aggregate loss $L$.

Let $\boldsymbol{Y}=\left(Y_{1}, \ldots, Y_{d}\right)^{\prime}$ have the stochastic representation $\boldsymbol{Y}=\sqrt{W} \boldsymbol{V}$ where $\boldsymbol{V}=\left(V_{1}, \ldots, V_{d}\right)^{\prime}$ is a vector of independent standard normal variables and $W$ is an independent positive random variable with a so-called generalized inverse Gaussian (GIG) distribution $W \sim N^{-}(\lambda, \chi, \kappa)$; see Formula (A1) in the Appendix A for the density of this distribution. The vector $Y$ has a spherical distribution $Y \sim S_{d}(\psi)$, and any component $Y$ has a univariate spherical distribution $Y \sim S_{1}(\psi)$, for a characteristic generator $\psi$ that depends on the particular choice of the parameters $\lambda, \chi$ and $\kappa$. An elliptical model of the kind described in Assumption 2(ii) is obtained by taking $X=A Y$ for $A \in \mathbb{R}^{d \times d}$ and satisfies $\boldsymbol{X} \sim E_{d}(\mathbf{0}, \Omega, \psi)$ where $\Omega=A A^{\prime} . \mathbf{X}$ is said to have a $d$-dimensional symmetric generalized hyperbolic $(\mathrm{GH})$ distribution.

To carry out our calculations it suffices to consider the single component $Y$. The variance of $Y$ satisfies $\operatorname{var}(Y)=\mathbb{E}(W)$ and an explicit formula for the case where $\chi>0$ and $\kappa>0$ is given in (A3). A formula for the characteristic function $\phi_{Y}$ is given in (A4) and the characteristic generator of the elliptical family can be inferred from the identity $\psi\left(s^{2}\right)=\phi_{Y}(s)$.

We consider four special one-parameter cases of this distribution resulting from particular choices of the parameters $\lambda, \chi$ and $\kappa$ of the GIG distribution:

1. The student $t$ distribution with degree of freedom $v$. This corresponds to the case where $\kappa=0$, $\lambda=-v / 2$ and $\chi=v$ or where $W$ has an inverse gamma distribution $W \sim \operatorname{IG}(v / 2, v / 2)$. In this case $\operatorname{var}(Y)=v /(v-2)$, provided $v>2$, and the characteristic function is given by (A5) in the Appendix A.

2. The variance gamma (VG) distribution. This corresponds to the case where $\chi=0$ or where $W$ has a gamma distribution $W \sim \mathrm{Ga}(\lambda, \kappa / 2)$. Without loss of generality we set the scaling parameter $\kappa=2$ so that $\operatorname{var}(Y)=\lambda$. The corresponding characteristic function is given by (A6).

3. The normal-inverse-Gaussian (NIG) distribution. This corresponds to the case where $\lambda=-1 / 2$. The distribution can be reparameterized in terms of $\theta=\sqrt{\chi^{\kappa}}$ and $\chi$; the latter parameter can be treated as a scaling parameter and set to one. The variance is then $\operatorname{var}(Y)=\theta^{-1}$ and the characteristic function is given by (A7).

4. The hyperbolic distribution. This corresponds to the case where $\lambda=1$. The distribution can be reparameterized in exactly the same way as the NIG distribution. The variance is $\operatorname{var}(Y)=\theta^{-1} K_{2}(\theta) / K_{1}(\theta)$ and the characteristic function is given by (A8).

\subsection{Summary of the Steps in the Calculation}

We return to the problem of calculating the scaling ratios $r_{\alpha}=c_{\alpha, \psi_{L}} / c_{\alpha, \psi_{1}}$ in (13) when the underlying risk-factor returns have symmetric distributions in the multivariate generalized hyperbolic family.

We recall the basic components that are required for the calculation: $Y \sim S_{1}(\psi)$ is spherically distributed with known standard deviation $\operatorname{sd}(Y)$ and known characteristic function $\phi_{Y}(s)=\psi\left(s^{2}\right)$; $Z \sim S_{1}\left(\psi_{1}\right)$ where $\psi_{1}=\psi^{h_{1}} ; L \sim S_{1}\left(\psi_{L}\right)$ where $\psi_{L}$ is given in (12). The steps are:

1. Calculate $\mathrm{ES}_{\alpha}(Z)$ using (18) and $\phi_{Z}(s)=\phi_{Y}^{h_{1}}(s)$.

2. Calculate $\operatorname{sd}(Z)=\sqrt{h_{1}} \operatorname{sd}(Y)$.

3. Hence calculate $c_{\alpha, \psi_{1}}=\mathrm{ES}_{\alpha}(Z) / \operatorname{sd}(Z)$.

4. Calculate $\mathrm{ES}_{\alpha}(L)$ using (18) and the fact that

$$
\phi_{L}(s)=\prod_{k=1}^{n} \phi_{Y}^{h_{k}-h_{k-1}}\left(s \sqrt{\boldsymbol{\beta}_{k}^{\prime} \Omega \boldsymbol{\beta}_{k}}\right) .
$$


5. Calculate $\operatorname{sd}(L)$ using the formula

$$
\operatorname{sd}(L)=\operatorname{sd}(Y) \sqrt{\sum_{k=1}^{n}\left(h_{k}-h_{k-1}\right) \beta_{k}^{\prime} \Omega \beta_{k}} .
$$

6. Hence calculate $c_{\alpha, \psi_{L}}=\mathrm{ES}_{\alpha}(L) / \operatorname{sd}(L)$.

7. Hence calculate the ratio $r_{\alpha}=c_{\alpha, \psi_{L}} / c_{\alpha, \psi_{1}}$.

\section{Results}

In the analyses of this section we make explicit choices of parametric distributions for the risk-factor changes in order to study the possible extent of risk overestimation that results from using the standard Basel liquidity formula. It is important to stress that most banks do not estimate parametric models for $\boldsymbol{X}_{t}$ in practice.

The vast majority of banks employ resampling techniques known as historical simulation. This means, effectively, that they estimate their models non-parametrically. It is certainly possible to fit multivariate hyperbolic distributions to data, even in high dimensions, using variants of the EM algorithm (McNeil et al. 2015; Protassov 2004), but we are not aware of banks that do this.

In the absence of data on the risk factors that affect a particular bank, we choose plausible values for the parameters of the generalized hyperbolic distributions by fitting univariate models to the broad market returns of the S\&P500 index. We also choose illustrative values for the elements of the matrix $\Omega$, since this matrix is not explicitly estimated by banks using the historical simulation method.

The values of the vectors $\beta_{k}$ depend on the sensitivities of the trading book positions to the risk factors. These would be known to a bank in practice. In the absence of data, we again make simple stylized choices.

\subsection{Design of Experiments}

In order to calibrate our distributions, we use 2132 observations of adjusted daily closing prices for the S\&P500 index, from 17 July 2007 to 31 December 2015, which have been converted to two-weekly log-returns (conforming approximately to 10 trading days, the base liquidity horizon required under FRTB).

We fit the various distributions discussed in Section 3.2 to the 10-day return data using the $\mathrm{R}$ package ghyp. Table 1 gives the estimated shape parameters for the distributions of interest; scale parameters are not required in our analysis. Note that we also confirm that the calculations for the Gaussian case yield a ratio of 1 , as a check on our implementation.

Table 1. Distribution parameters used in the calculation experiments. These have been derived by fitting these distributions to two-weekly log-returns of the S\&P500 index over the period from 17 July 2007 to 31 December 2015.

\begin{tabular}{lccc}
\hline Distribution Parameters & $\lambda$ & $\boldsymbol{\theta}$ & Remarks \\
\hline $\mathrm{t}$ & -1.46 & & $\nu=-2 \lambda$ \\
NIG & -0.5 & 0.49 & $\lambda$ fixed \\
Hyperbolic & 1 & 0.11 & $\lambda$ fixed \\
VG & 0.95 & & $\kappa=2$ \\
\hline
\end{tabular}

We carry out two experiments:

- In the first, we consider two risk factors, one in $B_{1}$ with a liquidity horizon of 10 days $\left(h_{1}=1\right)$ and the other in $B_{2}$ with a liquidity horizon of 20 days $\left(h_{2}=2\right)$. The dispersion matrix $\Omega$ is either taken to be the identity $\Omega=I_{2}$ (no correlation) or a correlation matrix with correlation $\rho=0.5$. 
- The second experiment follows in the same fashion but we assume there are 5 risk factors with liquidity horizons $10,20,40,60$ and 120 days $\left(h_{1}=1, h_{2}=2, h_{3}=4, h_{4}=6, h_{5}=12\right)$. We consider both the case where $\Omega=I_{5}$ and the case where $\Omega$ is an equicorrelation matrix with element $\rho=0.5$.

We present values of $c_{\alpha, \psi_{1}}, c_{\alpha, \psi_{L}}$ as well as the scaling ratio $r_{\alpha}$ for various confidence levels $\alpha$. The case of two risk factors is reported in Table 2 and the case of five risk factors is reported in Table 3.

Table 2. Constants $c_{\alpha, \psi_{1}}, c_{\alpha, \psi_{L}}$ and ratios $r_{\alpha}$ in the experiment with two risk factors.

\begin{tabular}{|c|c|c|c|c|c|c|c|}
\hline \multirow[b]{2}{*}{ Model } & \multirow{2}{*}{$\begin{array}{c}\alpha \\
\text { Quantity } \rho\end{array}$} & \multicolumn{2}{|c|}{0.95} & \multicolumn{2}{|c|}{0.975} & \multicolumn{2}{|c|}{0.99} \\
\hline & & 0 & 0.5 & 0 & 0.5 & 0 & 0.5 \\
\hline \multirow[t]{3}{*}{ Gauss } & $c_{\alpha, \psi_{1}}$ & 2.063 & 2.063 & 2.338 & 2.338 & 2.665 & 2.665 \\
\hline & $c_{\alpha, \psi_{L}}$ & 2.063 & 2.063 & 2.338 & 2.338 & 2.665 & 2.665 \\
\hline & $r_{\alpha}$ & 1.000 & 1.000 & 1.000 & 1.000 & 1.000 & 1.000 \\
\hline \multirow[t]{3}{*}{$\mathrm{t}$} & $c_{\alpha, \psi_{1}}$ & 2.223 & 2.223 & 2.906 & 2.906 & 4.065 & 4.065 \\
\hline & $c_{\alpha, \psi_{L}}$ & 2.212 & 2.169 & 2.831 & 2.671 & 3.868 & 3.486 \\
\hline & $r_{\alpha}$ & 0.995 & 0.975 & 0.974 & 0.919 & 0.952 & 0.858 \\
\hline \multirow[t]{3}{*}{ VG } & $c_{\alpha, \psi_{1}}$ & 2.345 & 2.345 & 2.841 & 2.841 & 3.509 & 3.509 \\
\hline & $c_{\alpha, \psi_{L}}$ & 2.247 & 2.132 & 2.670 & 2.468 & 3.225 & 2.891 \\
\hline & $r_{\alpha}$ & 0.958 & 0.909 & 0.940 & 0.869 & 0.919 & 0.824 \\
\hline \multirow[t]{3}{*}{ Hyperbolic } & $c_{\alpha, \psi_{1}}$ & 2.330 & 2.330 & 2.816 & 2.816 & 3.459 & 3.459 \\
\hline & $c_{\alpha, \psi_{L}}$ & 2.237 & 2.128 & 2.653 & 2.459 & 3.194 & 2.877 \\
\hline & $r_{\alpha}$ & 0.960 & 0.913 & 0.942 & 0.873 & 0.923 & 0.832 \\
\hline \multirow[t]{3}{*}{ NIG } & $c_{\alpha, \psi_{1}}$ & 2.374 & 2.374 & 2.976 & 2.976 & 3.832 & 3.832 \\
\hline & $c_{\alpha, \psi_{L}}$ & 2.296 & 2.167 & 2.801 & 2.544 & 3.502 & 3.042 \\
\hline & $r_{\alpha}$ & 0.967 & 0.913 & 0.941 & 0.855 & 0.914 & 0.794 \\
\hline
\end{tabular}

Table 3. Constants $c_{\alpha, \psi_{1}}, c_{\alpha, \psi_{L}}$ and ratios $r_{\alpha}$ in the experiment with five risk factors.

\begin{tabular}{|c|c|c|c|c|c|c|c|}
\hline \multirow[b]{2}{*}{ Model } & \multirow{2}{*}{$\begin{array}{c}\alpha \\
\text { Quantity } \rho\end{array}$} & \multicolumn{2}{|c|}{0.95} & \multicolumn{2}{|c|}{0.975} & \multicolumn{2}{|c|}{0.99} \\
\hline & & 0 & 0.5 & 0 & 0.5 & 0 & 0.5 \\
\hline \multirow[t]{3}{*}{ Gauss } & $c_{\alpha, \psi_{1}}$ & 2.063 & 2.063 & 2.338 & 2.338 & 2.665 & 2.665 \\
\hline & $c_{\alpha, \psi_{L}}$ & 2.063 & 2.063 & 2.338 & 2.338 & 2.665 & 2.665 \\
\hline & $r_{\alpha}$ & 1.000 & 1.000 & 1.000 & 1.000 & 1.000 & 1.000 \\
\hline \multirow[t]{3}{*}{$\mathrm{t}$} & $c_{\alpha, \psi_{1}}$ & 2.223 & 2.223 & 2.906 & 2.906 & 4.065 & 4.065 \\
\hline & $c_{\alpha, \psi_{L}}$ & 2.160 & 2.169 & 2.637 & 2.671 & 3.402 & 3.486 \\
\hline & $r_{\alpha}$ & 0.972 & 0.975 & 0.908 & 0.919 & 0.837 & 0.858 \\
\hline \multirow[t]{3}{*}{ VG } & $c_{\alpha, \psi_{1}}$ & 2.345 & 2.345 & 2.841 & 2.841 & 3.509 & 3.509 \\
\hline & $c_{\alpha, \psi_{L}}$ & 2.112 & 2.132 & 2.429 & 2.468 & 2.824 & 2.891 \\
\hline & $r_{\alpha}$ & 0.901 & 0.909 & 0.855 & 0.869 & 0.805 & 0.824 \\
\hline \multirow[t]{3}{*}{ Hyperbolic } & $c_{\alpha, \psi_{1}}$ & 2.330 & 2.330 & 2.816 & 2.816 & 3.459 & 3.459 \\
\hline & $c_{\alpha, \psi_{L}}$ & 2.108 & 2.128 & 2.423 & 2.459 & 2.814 & 2.877 \\
\hline & $r_{\alpha}$ & 0.905 & 0.913 & 0.860 & 0.873 & 0.813 & 0.832 \\
\hline \multirow[t]{3}{*}{ NIG } & $c_{\alpha, \psi_{1}}$ & 2.374 & 2.374 & 2.976 & 2.976 & 3.832 & 3.832 \\
\hline & $c_{\alpha, \psi_{L}}$ & 2.142 & 2.167 & 2.492 & 2.544 & 2.942 & 3.042 \\
\hline & $r_{\alpha}$ & 0.902 & 0.913 & 0.837 & 0.855 & 0.768 & 0.794 \\
\hline
\end{tabular}

\subsection{Results}

In both tables it is clear that the scaling ratios are less than one for all non-Gaussian cases meaning that the Basel liquidity formula is indeed conservative when the risk factors have a multivariate elliptical distribution from one of the four generalized hyperbolic sub-families considered in Section 3.2 and Table 1. 
The second experiment with five liquidity buckets leads in general to smaller values for the scaling ratios than the first experiment with two buckets. Thus the degree of conservatism of the formula increases with the number of liquidity buckets. This is in line with the increase in the central limit effect as we aggregate over more time periods.

Introducing correlation leads to an increase in the constants $c_{\alpha, \psi_{L}}$ and hence an increase in the scaling ratio. In other words, the weaker the correlation, the more conservative the liquidity formula. To understand why this is the case, note that the constants $c_{\alpha, \psi_{L}}$ depend on the characteristic generator $\psi_{L}$ in (12) and hence on the set of values $\left\{\boldsymbol{\beta}_{k}^{\prime} \Omega \boldsymbol{\beta}_{k}, k=1, \ldots, n\right\}$. By considering Formula (4) we can think of these as the relative weights attached to each of the $n$ liquidity buckets. When $\rho=0$ these weights are $(5,4,3,2,1)$ but when $\rho=0.5$ they are $(15,10,6,3,1)$. The intuition is that, in the second case, the first few liquidity buckets dominate more in the convolution calculation and the central limit effect is mitigated.

Considering the different generalized hyperbolic special cases we see that the ratios are usually largest for the $t$ distribution followed by the other three distributions; the exact ordering depends on the confidence level $\alpha$ used in the calculation. In other words, use of the Basel liquidity formula is least conservative in the case of $t$ and more conservative for the other distributions.

When we look at the confidence level of $\alpha=0.975$ which is the level used in the new capital standard (Basel Committee on Banking Supervision 2016) the normal inverse Gaussian (NIG) distribution leads to the highest level of conservatism. This distribution is often a plausible model in market risk applications. The ratio in the case where $n=5$ and $\rho=0$ is 0.837 which means that the Basel liquidity formula would tend to overstate capital by around $19.4 \%$.

It would be appealing to link the values of $r_{\alpha}$ and the resulting levels of conservatism of the Basel formula to some parameter that describes the heavy-tailedness of the distributions under consideration, such as their tail index or kurtosis. However the only distribution in Tables 2 and 3 which has a regularly varying tail, and thus a finite tail index, is the t distribution. Although the VG, hyperbolic and NIG distributions have infinite tail indices, they tend to give smaller ratios $r_{\alpha}$. Moreover the $t$ distribution has infinite kurtosis while the other distributions have finite kurtosis. It would seem that there is a more complex story behind the precise ordering of the $r_{\alpha}$ values. However, the results are sufficient to show that a range of differing heavy-tailed distributions all lead to ratios less than one.

\section{Conclusions}

We have presented evidence that the Basel liquidity formula tends to lead to conservative capital charges when financial risk factors come from heavier-tailed elliptical distributions.

The Basel formula is clearly a heavily stylized formula and makes a number of crude assumptions. We have concentrated on the effect of changing the underlying distribution of the risk factors when portfolio sensitivities are linear. However, there are other important effects we have not considered which will have an influence on the ability of the formula to capture risk. In particular, the true effect of risk-factor changes on portfolio risk is likely to be highly non-linear over the kind of time horizons we consider. Moreover, as we have already noted, positive serial correlation between losses over different sub-intervals $\left[h_{k-1}, h_{k}\right]$ of the overall liquidity horizon $\left[0, h_{n}\right]$ will tend to lead to a tendency towards underestimation which may counteract the central limit effect.

We note that our assumption that risk-factor changes are elliptically distributed implies that their marginal distributions are symmetric. This is clearly a limiting assumption and it would be of interest to see if the liquidity formula could be further generalized to classes of distribution that admit skewness, such as the full generalized hyperbolic family.

In writing about inherent conservatism in the liquidity formula we are well aware that there are many further layers of conservatism built into the new system of risk charges for the trading book, such as the requirement to calibrate the model to stress periods and the requirement to adjust the calculation to understate the possible diversification effects across risk factors. These other features may have greater impact than the issue we address. 
Nonetheless it is important to be clear about the workings of the formula and the extent to which it may be interpreted as a principles-based approach to the measurement of market risk. Our study should be understood as a contribution to the clarification of this issue.

Author Contributions: Conceptualization, A.J.M.; Formal analysis, J.B. and A.J.M.; Methodology, J.B. and A.J.M.; Software, J.B. and A.J.M.; Writing, J.B. and A.J.M.

Funding: This research received no external funding.

Conflicts of Interest: The authors declare no conflict of interest.

\section{Appendix A}

A standardized univariate generalized hyperbolic random variable $Y$ has the stochastic representation $Y=\sqrt{W} V$ where $V$ is a standard normal variable and $W$ is an independent positive random variable with a generalized-inverse-Gaussian (GIG) distribution. The density of the latter is

$$
f_{W}(w)=\frac{\chi^{-\lambda}(\sqrt{\chi \kappa})^{\lambda}}{2 K_{\lambda}(\sqrt{\chi \kappa})} w^{\lambda-1} \exp \left(-\frac{1}{2}\left(\chi w^{-1}+\kappa w\right)\right), \quad \begin{cases}\chi>0, \kappa \geq 0 & \text { if } \lambda<0 \\ \chi>0, \kappa>0 & \text { if } \lambda=0 \\ \chi \geq 0, \kappa>0 & \text { if } \lambda>0\end{cases}
$$

where $K_{\lambda}$ denotes a Bessel function of the third kind. The characteristic function of $Y$ is given by

$$
\phi_{Y}(s)=\mathbb{E}(\mathbb{E}(\exp (i s \sqrt{W} V) \mid W))=\mathbb{E}\left(\exp \left(-\frac{1}{2} s^{2} W\right)\right)=\int_{0}^{\infty} e^{-\frac{1}{2} s^{2} w} f_{W}(w) \mathrm{d} w
$$

and the variance by $\operatorname{var}(Y)=\mathbb{E}(W)$.

We first consider the case where $\chi>0$ and $\kappa>0$. In this case the variance of $Y$ is

$$
\operatorname{var}(Y)=\left(\frac{\chi}{\kappa}\right)^{1 / 2} \frac{K_{\lambda+1}\left(\sqrt{\chi^{\kappa}}\right)}{K_{\lambda}\left(\sqrt{\chi^{\kappa}}\right)}
$$

and the characteristic function is

$$
\begin{aligned}
\phi_{Y}(s) & =\int_{0}^{\infty} e^{-\frac{1}{2}\left(\chi w^{-1}+\left(s^{2}+\kappa\right) w\right)} \frac{\chi^{-\lambda}(\chi \kappa)^{\lambda / 2}}{2 K_{\lambda}(\sqrt{\chi \kappa})} x^{\lambda-1} \mathrm{~d} w \\
& =\left(\frac{\kappa}{s^{2}+\kappa}\right)^{\lambda / 2} \frac{K_{\lambda}\left(\sqrt{\chi\left(s^{2}+\kappa\right)}\right)}{K_{\lambda}\left(\sqrt{\chi^{\kappa}}\right)} .
\end{aligned}
$$

We next consider the case of a Student $t$ distribution which corresponds to $\kappa=0, \lambda=-v / 2$ and $\chi=v$. In this case $W$ has an inverse gamma distribution $W \sim \operatorname{IG}(v / 2, v / 2)$ and $\operatorname{var}(Y)=\mathbb{E}(W)=$ $v /(v-2)$, provided $v>2$. The characteristic function should be interpreted as the limit of (A4) as $\kappa \rightarrow 0$. Substituting the density of an inverse gamma distribution into (A2) yields

$$
\begin{aligned}
\phi_{Y}(s) & =\int_{0}^{\infty} e^{-\frac{1}{2} s^{2} w} \frac{\left(\frac{1}{2} v\right)^{v / 2}}{\Gamma\left(\frac{1}{2} v\right)} w^{-\frac{v}{2}-1} e^{-\frac{1}{2} v w^{-1}} \mathrm{~d} w \\
& =\frac{\left(v s^{2}\right)^{v / 4}}{2^{v / 2-1} \Gamma\left(\frac{1}{2} v\right)} K_{v / 2}\left(\sqrt{v s^{2}}\right)
\end{aligned}
$$

The special case of variance gamma (VG) corresponds to $\chi=0$; without loss of generality we set the scaling parameter $\kappa=2$. In this case $W$ has a gamma distribution $W \sim \operatorname{Ga}(\lambda, 1)$ and $\operatorname{var}(Y)=\mathbb{E}(W)=\lambda$. The characteristic function in this case should be interpreted as the limit of (A4) as $\chi \rightarrow 0$. Substituting the density of a gamma distribution $W \sim \mathrm{Ga}(\lambda, 1)$ for $f_{W}$ in (A2) we obtain 


$$
\begin{aligned}
\phi_{Y}(s) & =\int_{0}^{\infty} e^{-\frac{1}{2} s^{2} w} \frac{w^{\lambda-1} e^{-w}}{\Gamma(\lambda)} \mathrm{d} w \\
& =\left(1+\frac{1}{2} s^{2}\right)^{-\lambda} .
\end{aligned}
$$

Two further special cases are the normal inverse Gaussian (NIG) and hyperbolic distributions. In both cases we fix the parameter $\lambda$ and reparameterize the GH distribution in terms of $\theta=\sqrt{\chi^{\kappa}}$ and $\kappa$; the latter then appears only as a scaling parameter and can be set to one.

For the NIG distribution $\lambda=-1 / 2$ and $\operatorname{var}(Y)=\theta^{-1}$. The identity $K_{\lambda}(x)=K_{-\lambda}(x)$ can be used to infer that

$$
\phi_{Y}(s)=\left(\frac{\sqrt{\theta^{2}+s^{2}}}{\theta}\right)^{1 / 2} \frac{K_{1 / 2}\left(\sqrt{\theta^{2}+s^{2}}\right)}{K_{1 / 2}(\theta)} .
$$

For the hyperbolic distribution $\lambda=1$ and $\operatorname{var}(Y)=\theta^{-1} K_{2}(\theta) / K_{1}(\theta)$. The characteristic function is

$$
\phi_{Y}(s)=\left(\frac{\theta}{\sqrt{\theta^{2}+s^{2}}}\right) \frac{K_{1}\left(\sqrt{\theta^{2}+s^{2}}\right)}{K_{1}(\theta)} .
$$

\section{References}

Barndorff-Nielsen, Ole. 1978. Hyperbolic distributions and distributions on hyperbolae. Scandinavian Journal of Statistics 5: 151-57.

Barndorff-Nielsen, Ole, and Preben Blaesild. 1981. Hyperbolic distributions and ramifications: Contributions to theory and application. In Statistical Distributions in Scientific Work. Edited by Taillie Charles, Ganapati P. Patil and Bruno A. Baldessari. Dordrecht: Reidel, vol. 4, pp. 19-44.

Basel Committee on Banking Supervision. 2013. Fundamental Review of the Trading Book: A Revised Market Risk Framework. Publication No. 265. Basel: Bank of International Settlements.

Basel Committee on Banking Supervision. 2016. Minimum Capital Requirements for Market Risk. Publication No. 352. Basel: Bank of International Settlements.

Nesmith, Travis D., and Dong Hwan Oh. 2017. Accurate evaluation of expected shortfall for linear portfolios with elliptically distributed risk factors. Journal of Risk and Financial Management 10: 5.

Eberlein, Ernst. 2010. Generalized hyperbolic models. In Encyclopedia of Quantitative Finance. Edited by Rama Cont. New York: Wiley, pp. 833-36.

Fang, Kai Wang, Samuel Kotz, and Kai Wang Ng. 1990. Symmetric Multivariate and Related Distributions. London: Chapman \& Hall.

Gil-Pelaez, J. 1951. Note on the inversion theorem. Biometrika 38: 481-82. Available online: https:/ /academic.oup. $\mathrm{com} /$ biomet/article-pdf/38/3-4/481/718851/38-3-4-481.pdf (accessed on 3 July 2018).

Kamdem, Jules Sadefo. 2005. Value-at-risk and expected shortfall for linear portfolios with elliptically distributed risk factors. International Journal of Theoretical and Applied Finance 8: 537-51. [CrossRef]

McNeil, Alexander J., Rüdiger Frey, and Paul Embrechts. 2015. Quantitative Risk Management: Concepts, Techniques and Tools, 2nd ed. Princeton: Princeton University Press.

Podgórski, Krzysztof, and Jonas Wallin. 2016. Convolution-invariant subclasses of generalized hyperbolic distributions. Communications in Statistics-Theory and Methods 45: 98-103. [CrossRef]

Protassov, Rostislav S. 2004. EM-based maximum likelihood parameter estimation of multivariate generalized hyperbolic distributions with fixed $\lambda$. Statistics and Computing 14: 67-77. [CrossRef]

(C) 2018 by the authors. Licensee MDPI, Basel, Switzerland. This article is an open access article distributed under the terms and conditions of the Creative Commons Attribution (CC BY) license (http://creativecommons.org/licenses/by/4.0/). 